TRANSACTIONS OF THE

AMERICAN MATHEMATICAL SOCIETY

Volume 355, Number 7, Pages 2663-2674

S 0002-9947(03)03194-5

Article electronically published on February 27, 2003

\title{
TAYLOR EXPANSION OF AN EISENSTEIN SERIES
}

\author{
TONGHAI YANG
}

ABstract. In this paper, we give an explicit formula for the first two terms of the Taylor expansion of a classical Eisenstein series of weight $2 k+1$ for $\Gamma_{0}(q)$. Both the first term and the second term have interesting arithmetic interpretations. We apply the result to compute the central derivative of some Hecke $L$-functions.

\section{Introduction}

Consider the classical Eisenstein series

$$
\sum_{\gamma \in \Gamma_{\infty} \backslash \mathrm{SL}_{2}(\mathbb{Z})} \operatorname{Im}(\gamma \tau)^{s},
$$

which has a simple pole at $s=1$. The well-known Kronecker limit formula gives a closed formula for the next term (the constant term) in terms of the Dedekind $\eta$-function and has a lot of applications in number theory. It seems natural and worthwhile to study the same question for more general Eisenstein series. For example, consider the Eisenstein series

$$
E(\tau, s)=\sum_{\gamma \in \Gamma_{\infty} \backslash \Gamma_{0}(q)} \epsilon(d)(c \tau+d)^{-2 k-1} \operatorname{Im}(\gamma \tau)^{\frac{s}{2}-k} .
$$

Here $\gamma=\left(\begin{array}{ll}a & b \\ c & d\end{array}\right),-q$ is a fundamental discriminant of an imaginary quadratic field, and $\epsilon=\left(\frac{-q}{}\right)$. This Eisenstein series was used in the celebrated work of Gross and Zagier (GZ, Chapter IV]) to compute the central derivative of cuspidal modular forms of weight $2 k+2$. The Eisenstein series is holomorphic (as a function of $s$ ) at the symmetric center $s=0$ with the leading term (constant term) given by a theta series via the Siegel-Weil formula. The analogue of the Kronecker limit formula would be a closed formula for the central derivative at $s=0$ - the main object of this paper. This would give a direct proof of [GZ] Proposition 4.5]. Another application is to give a closed formula for the central derivative of a family of Hecke $L$-series associated to CM abelian varieties, which is very important in the arithmetic of CM abelian varieties in view of the Birch and Swinnerton-Dyer conjecture. This application will be given in section 4 . We will also prove a transformation equation for the tangent line of the Eisenstein series at the center, which should be of independent interest.

Received by the editors September 9, 2002.

2000 Mathematics Subject Classification. Primary 11G05, 11M20, 14H52.

Key words and phrases. Kronecker formula, central derivative, elliptic curves, Eisenstein series. Partially supported by an AMS Centennial fellowship and NSF grant DMS-0070476. 
To make the exposition simple, we assume that $q>3$ is a prime congruent to 3 modulo 4. Let $\boldsymbol{k}=\mathbb{Q}(\sqrt{-q})$.

Set

$$
\Lambda(s, \epsilon)=\pi^{-\frac{s+1}{2}} \Gamma\left(\frac{s+1}{2}\right) L(s, \epsilon)
$$

and

$$
E^{*}(\tau, s)=q^{\frac{s+1}{2}} \Lambda(s+1, \epsilon) E(\tau, s) .
$$

It is well known that $E^{*}(\tau, s)$ is holomorphic.

As in [GZ, Propositions 4.4 and 3.3], we define

$$
p_{k}(t)=\sum_{m=0}^{k}\left(\begin{array}{l}
k \\
m
\end{array}\right) \frac{(-t)^{m}}{m !}
$$

and

$$
q_{k}(t)=\int_{1}^{\infty} e^{-t u}(u-1)^{k} u^{-k-1} d u, \quad t>0 .
$$

We remark that $p_{k}(-t)$ and $q_{k}(t)$ are two "basic" solutions of the differential equations

$$
t C^{\prime \prime}(t)+(1+t) C^{\prime}(t)-k C(t)=0 .
$$

Finally, let $\rho(n)$ be given by

$$
\zeta_{\boldsymbol{k}}(s)=\sum \rho(n) n^{-s} .
$$

Theorem 0.1. Let the notation be as above, and let $h$ be the ideal class number of $\boldsymbol{k}$. Write $\tau=u+i v$. Then

$$
E^{*}(\tau, 0)=v^{-k}\left(h+2 \sum_{n>0} \rho(n) p_{k}(4 \pi n v) e(n \tau)\right)
$$

and

$$
\begin{aligned}
& E^{* \prime}(\tau, 0)+\frac{1}{4} \sum_{j=1}^{k} \frac{1}{j} E^{*}(\tau, 0) \\
& =\frac{1}{2} v^{-k}\left[a_{0}(v)-2 \sum_{n>0} a_{n} p_{k}(4 \pi n v) e(n \tau)-2 \sum_{n<0} \rho(-n) q_{k}(-4 \pi n v) e(n \tau)\right] .
\end{aligned}
$$

Here

and

$$
a_{0}(v)=h\left(\log (q v)+2 \frac{\Lambda^{\prime}(1, \epsilon)}{\Lambda(1, \epsilon)}+\sum_{j=1}^{k} \frac{1}{j}\right)
$$

$$
a_{n}=\left(\operatorname{ord}_{q} n+1\right) \rho(n) \log q+\sum_{\left(\frac{p}{q}\right)=-1}\left(\operatorname{ord}_{p} n+1\right) \rho(n / p) \log p .
$$

The formulas should be compared to those for $\tilde{\Phi}$ in [GZ, Propositions 4.4 and 4.5]. In fact, multiplying our formulas by the theta function in their paper and taking the trace would yield their formulas for $\tilde{\Phi}$. The method used here seems to be more suitable for generalization. The proof is based on the observation that the Eisenstein series (0.1) can be split into two Eisenstein series. One of them is coherent, and it is easy to compute its value. It contributes little to the central 
derivative. The other one is incoherent, contributes nothing to the value, and its central derivative can be computed by the method of [KRY], where we dealt with the case $k=0$. This consists of sections 1 and 2 .

In section 3, we study how the value and derivative behave under the Fricke involution $\tau \mapsto-1 / q \tau$ and obtain the following functional equation. One interesting point about the equation is that it basically follows from the definition of automorphic forms (see (3.2)).

Theorem 0.2. The modular forms $E^{*}(\tau, 0)$ and $E^{* \prime}(\tau, 0)$ satisfy the following functional equation:

$$
\left(\begin{array}{l}
E^{*}\left(-\frac{1}{q \tau}, 0\right) \\
E^{* \prime}\left(-\frac{1}{q \tau}, 0\right)
\end{array}\right)=i(\sqrt{q} \tau)^{2 k+1}\left(\begin{array}{cc}
-1 & 0 \\
\sum_{j=1}^{k} \frac{1}{j}+\frac{1}{2} \log q & 1
\end{array}\right)\left(\begin{array}{l}
E^{*}(\tau, 0) \\
E^{* \prime}(\tau, 0)
\end{array}\right) .
$$

Finally, let $\mu$ be a canonical Hecke character of weight 1 of $\boldsymbol{k}$ (see section 4 for the definition). It is associated to the CM elliptic curve $A(q)$ studied by Gross (Gro]). When $q \equiv 3 \bmod 8$, S. Miller and the author proved recently that the central derivative $L^{\prime}(1, \mu) \neq 0$ ([MY]). Since the central derivative encodes very important information in the arithmetic of $A(q)$, it is important to find a good formula for the central derivative. Standard calculation shows that the $L$-series $L(s, \mu)$ is $E(\tau, 2 s)$ evaluated at a CM cycle. So Theorem 0.1 gives an explicit formula for the central derivative $L^{\prime}(1, \mu)$ (Corollary 4.2).

\section{Coherent And incoherent Eisenstein SERIES}

Let $G=\mathrm{SL}_{2}$ over $\mathbb{Q}$, and let $B=T N$ be the standard Borel subgroup, where $T$ is the standard maximal split torus of $B$ and $N$ is the unipotent radical of $B$. Their rational points are given by

$$
T(\mathbb{Q})=\left\{m(a)=\left(\begin{array}{cc}
a & 0 \\
0 & a^{-1}
\end{array}\right): a \in \mathbb{Q}^{*}\right\}
$$

and

$$
N(\mathbb{Q})=\left\{n(b)=\left(\begin{array}{ll}
1 & b \\
0 & 1
\end{array}\right): b \in \mathbb{Q}\right\} .
$$

Consider the global induced representation

$$
I(s, \epsilon)=\left.\operatorname{Ind}_{B(\mathbb{A})}^{G(\mathbb{A})} \epsilon\right|_{\mathbb{A}} ^{s}
$$

of $G(\mathbb{A})$, where $\mathbb{A}$ is the ring of adèles of $\mathbb{Q}$. By definition a section $\Phi(s) \in I(s, \epsilon)$ satisfies

$$
\Phi(n(b) m(a) g, s)=\epsilon(a)|a|^{s+1} \Phi(g, s)
$$

for $a \in \mathbb{A}^{*}$ and $b \in \mathbb{A}$. Let $K=\mathrm{SL}_{2}(\hat{\mathbb{Z}})$ and let $K_{\infty}=\mathrm{SO}(2)(\mathbb{R})$. Associated to a standard section $\Phi$, which means that its restriction on $K K_{\infty}$ is independent of $s$, one defines the Eisenstein series

$$
E(g, s, \Phi)=\sum_{\gamma \in B(\mathbb{Q}) \backslash G(\mathbb{Q})} \Phi(\gamma g, s) .
$$

It is absolutely convergent for $\operatorname{Re} s>1$ and has a meromorphic continuation to the whole complex $s$-plane. We consider three standard sections $\Phi^{0}, \Phi^{ \pm}$in this paper. For every prime $p \nmid q \infty$, let $\Phi_{p} \in I\left(s, \epsilon_{p}\right)$ be the unique spherical section 
such that $\Phi_{p}(x)=1$ for every $x \in K_{p}=\mathrm{SL}_{2}\left(\mathbb{Z}_{p}\right)$. Let $\Phi_{\infty} \in I\left(s, \epsilon_{\infty}\right)$ be the unique section of weight $2 k+1$ in the sense that

$$
\Phi_{\infty}\left(g k_{\theta}, s\right)=\Phi_{\infty}(g, s) e^{i(2 k+1) \theta}
$$

for every $k_{\theta}=\left(\begin{array}{cc}\cos \theta & \sin \theta \\ -\sin \theta & \cos \theta\end{array}\right) \in K_{\infty}$. For $p=q$, let

$$
J_{q}=\left\{\left(\begin{array}{cc}
a & b \\
c q & d
\end{array}\right) \in S L_{2}\left(\mathbb{Z}_{q}\right): a, b, c, d \in \mathbb{Z}_{q}\right\}
$$

be the Iwahori subgroup of $K_{q}$. Then $\epsilon_{q}$ defines a character of $J_{q}$ via

$$
\epsilon_{q}\left(\left(\begin{array}{cc}
a & b \\
c q & d
\end{array}\right)\right)=\epsilon_{q}(d) .
$$

As described in [KRY, section 2], the subspace of $I\left(s, \epsilon_{q}\right)$ consisting of $\epsilon_{q}$ eigenvectors of $J_{q}$ is two-dimensional and is spanned by the cell functions of $\Phi_{q}^{i}$, determined by

$$
\Phi_{q}^{i}\left(w_{j}, s\right)=\delta_{i j}, \quad \text { where } w_{0}=1 \text { and } w_{1}=w=\left(\begin{array}{cc}
0 & -1 \\
1 & 0
\end{array}\right) .
$$

We denote this subspace by $W\left(J_{q}, \epsilon_{q}, s\right)$. A better basis for this subspace turns out to be given by

$$
\Phi_{q}^{ \pm}=\Phi_{q}^{0} \pm \frac{1}{\sqrt{-q}} \Phi_{q}^{1},
$$

which are "eigenfunctions" of some intertwining operator (see Lemma 2.2). Set

$$
\Phi^{0}=\Phi_{q}^{0} \prod_{p \neq q} \Phi_{p} \quad \text { and } \quad \Phi^{ \pm}=\Phi_{q}^{ \pm} \prod_{p \neq q} \Phi_{p} .
$$

Clearly, $\Phi^{0}=\frac{1}{2}\left(\Phi^{+}+\Phi^{-}\right)$. For $\tau=u+i v$ with $v>0$, let

$$
g_{\tau}=n(u) m(\sqrt{v}) .
$$

Then standard computation gives

Proposition 1.1. Let the notation be as above. Then

$$
\begin{aligned}
E^{*}(\tau, s) & =v^{-k-\frac{1}{2}} E^{*}\left(g_{\tau}, s, \Phi^{0}\right) \\
& =\frac{1}{2} v^{-k-\frac{1}{2}}\left(E^{*}\left(g_{\tau}, s, \Phi^{+}\right)+E^{*}\left(g_{\tau}, s, \Phi^{-}\right)\right) .
\end{aligned}
$$

Here

$$
E^{*}(g, s, \Phi)=q^{\frac{s+1}{2}} \Lambda(s+1, \epsilon) E(g, s, \Phi)
$$

is the completion of the Eisenstein series $E(g, s, \Phi)$.

As we will see in Proposition 2.4, the Eisenstein series with $\Phi^{ \pm}$behave almost as "even/odd" functions respectively, and both have nice functional equations. This is not a coincidence. Indeed, from the point of view of representation theory, $\Phi^{+}(g, 0)$ is a coherent section in $I(0, \epsilon)$ in the sense that it comes from a global (two-dimensional) quadratic space, while $\Phi^{-}(g, 0)$ is an incoherent section in $I(0, \epsilon)$, coming from a collection of inconsistent local quadratic spaces. We refer to $\mathrm{Ku}$ for explanation of this terminology and for a general idea for computing the central derivative of incoherent Eisenstein series. Every section in $I(0, \epsilon)$ is a linear combination of coherent and incoherent sections; we just made it explicit in this case. 


\section{Proof of Theorem 0.1}

Let $\psi=\prod \psi_{p}$ be the "canonical" additive character of $\mathbb{A}$ via

$$
\psi_{p}(x)= \begin{cases}e^{2 \pi i x} & \text { if } p=\infty, \\ e^{-2 \pi i \lambda(x)} & \text { if } p \neq \infty .\end{cases}
$$

Here $\lambda$ is the canonical map $\mathbb{Q}_{p} \longrightarrow \mathbb{Q}_{p} / \mathbb{Z}_{p} \hookrightarrow \mathbb{Q} / \mathbb{Z}$. For a standard section $\Phi=\prod \Phi_{p} \in I(s, \epsilon)$ and $d \in \mathbb{Q}$, one defines the local Whittaker function

$$
W_{d, p}(g, s, \Phi)=\int_{\mathbb{Q}_{p}} \Phi(w n(b) g, s) \psi_{p}(-d b) d b .
$$

Let

$$
W_{d, p}^{*}(g, s, \Phi)=L_{p}(s+1, \epsilon) W_{d, p}(g, s, \Phi)
$$

be its completion. We also set $M_{p}(s)=W_{0, p}(s)$ and $M_{p}^{*}(s)=W_{0, p}^{*}(s)$. So $M^{*}(s)=$ $\prod M_{p}^{*}(s)$ is a normalized intertwining operator from $I(s, \epsilon)$ to $I(-s, \epsilon)$.

In general, an Eisenstein series $E^{*}(g, s, \Phi)$ has a Fourier expansion

$$
E^{*}(g, s, \Phi)=\sum_{d} E_{d}^{*}(g, s, \Phi)
$$

with

$$
E_{d}^{*}(g, s, \Phi)=q^{\frac{s+1}{2}} \prod_{p} W_{d, p}^{*}(g, s, \Phi)
$$

for $d \neq 0$ and

$$
E_{0}^{*}(g, s, \Phi)=q^{\frac{s+1}{2}} \Lambda(s+1, \epsilon) \Phi(g, s)+q^{\frac{s+1}{2}} M^{*}(s) \Phi(g, s) .
$$

The local Whittaker integrals are computed in the next three lemmas.

Lemma 2.1 ([KRY, Lemma 2.4]). For a finite prime number $p \neq q$, one has $W_{d, p}^{*}\left(1, s, \Phi_{p}\right)=0$ unless $\operatorname{ord}_{p} d \geq 0$. In such a case, one has

$$
W_{d, p}^{*}\left(1, s, \Phi_{p}\right)=\sum_{r=0}^{\operatorname{ord}_{p} d}\left(\epsilon_{p}(p) p^{-s}\right)^{r}
$$

and

$$
M_{p}^{*}(s) \Phi(s)=L_{p}(s, \epsilon) \Phi_{p}(-s) .
$$

Here $\Phi_{p}$ is the unique spherical section defined in section 1. In particular,

$$
W_{d, p}^{*}\left(1,0, \Phi_{p}\right)=\rho_{p}(d),
$$

where $\rho_{p}(d)=\rho\left(p^{\operatorname{ord}_{p} d}\right)$ for $p<\infty$.

Lemma 2.2. For $p=q$, one has

$$
\left(\begin{array}{l}
W_{d, q}^{*}\left(w_{0}, s, \Phi^{ \pm}\right) \\
W_{d, q}^{*}\left(w_{1}, s, \Phi^{ \pm}\right)
\end{array}\right)= \begin{cases}\left(1 \pm \epsilon_{q}(d) q^{-s\left(\operatorname{ord}_{q} d+1\right)}\right)\left(\begin{array}{c} 
\pm \frac{1}{\sqrt{-q}} \\
-\frac{1}{q}
\end{array}\right) & \text { if } \operatorname{ord}_{q} d \geq 0, \\
\left(1 \pm \epsilon_{q}(d)\right)\left(\begin{array}{c}
0 \\
-q^{-1}
\end{array}\right) & \text { if } \operatorname{ord}_{q} d=-1, \\
0 & \text { otherwise }\end{cases}
$$


and

$$
M_{q}^{*}(s) \Phi_{q}^{ \pm}= \pm \frac{1}{\sqrt{-q}} \Phi_{q}^{ \pm} .
$$

Proof. The first formula follows from [KRY, (3.26)-(3.29)]. For the second formula, notice that $M_{q}^{*}(s)$ is an intertwining operator between eigenspaces $W\left(J_{q}, \epsilon_{q}, s\right)$ and $W\left(J_{q}, \epsilon_{q},-s\right)$ of $J_{p}$. So

$$
M_{q}^{*}(s) \Phi_{q}^{ \pm}=a^{ \pm} \Phi_{q}^{+}+b^{ \pm} \Phi_{q}^{-}
$$

for some constants $a^{ \pm}$and $b^{ \pm}$. Plugging in $g=w_{0}$ and $w_{1}$, and applying the first formula, one gets the desired formula.

Lemma 2.3. Let $\Phi=\Phi_{\infty}$ be the local section in $I\left(s, \epsilon_{\infty}\right)$ defined by (1.3).

(1)

$$
W_{d, \infty}^{*}\left(g_{\tau}, s, \Phi\right)=2 i v^{\frac{1+s}{2}} \pi^{-\frac{s}{2}} e(d u) \prod_{j=0}^{k} \frac{j-\frac{s}{2}}{j+\frac{s}{2}} \frac{\eta\left(2 v, \pi d, \frac{s}{2}+k+1, \frac{s}{2}-k\right)}{\Gamma\left(\frac{s}{2}\right)} .
$$

Here

$$
\eta(g, h, \alpha, \beta)=\int_{x \pm h>0} e^{-g x}(x+h)^{\alpha-1}(x-h)^{\beta-1} d x
$$

is Shimura's eta function for $g>0, h \in \mathbb{R}$, and $\operatorname{Re} \alpha$ and $\operatorname{Re} \beta$ sufficiently large Sh.

(2) For $d>0$, one has

$$
W_{d, \infty}^{*}\left(g_{\tau}, 0, \Phi\right)=2 i v^{\frac{1}{2}} p_{k}(4 \pi d v) e(d \tau),
$$

where $p_{k}$ is defined by (0.4).

(3) For $d<0$, one has $W_{d, \infty}^{*}\left(g_{\tau}, 0, \Phi\right)=0$, and

$$
W_{d, \infty}^{* \prime}\left(g_{\tau}, 0, \Phi\right)=i v^{\frac{1}{2}} q_{k}(-4 \pi d v) e(d \tau),
$$

where $q_{k}$ is given by $(0.5)$.

(4) $\quad M_{\infty}^{*}(s) \Phi_{\infty}(s)=i \prod_{j=0}^{k} \frac{j-s / 2}{j+s / 2} L_{\infty}(s, \epsilon) \Phi_{\infty}(-s)$.

Proof. The proof is the same as that of [KRY, Proposition 2.6] and is left to the reader.

Proposition 2.4. One has the functional equation as $s$ goes to $-s$ :

$$
\prod_{j=0}^{k}\left(j-\frac{s}{2}\right) E^{*}\left(g,-s, \Phi^{ \pm}\right)= \pm \prod_{j=0}^{k}\left(j+\frac{s}{2}\right) E^{*}\left(g, s, \Phi^{ \pm}\right) .
$$

Proof. By Lemmas 2.1-2.3, one has

$$
M^{*}(s) \Phi(g, s)= \pm q^{-\frac{1}{2}} \prod_{j=0}^{k} \frac{j-\frac{s}{2}}{j+\frac{s}{2}} \Lambda(s, \epsilon) \Phi(g,-s) .
$$

Now the proposition follows from the functional equations

$$
q^{\frac{s}{2}} \Lambda(s, \epsilon)=q^{-\frac{s}{2}} \Lambda(-s, \epsilon)
$$

and

$$
E(g, s, \Phi)=E(g,-s, M(s) \Phi) .
$$

Here $M(s)=M^{*}(s) \Lambda(s+1, \epsilon)^{-1}$ is the unnormalized intertwining operator from $I(s, \epsilon)$ to $I(-s, \epsilon)$. 
Theorem 2.5. One has

$$
v^{-\frac{1}{2}} E^{*}\left(g_{\tau}, 0, \Phi^{+}\right)=2\left(h_{q}+2 \sum_{n>0} \rho(n) p_{k}(4 \pi n v) e(n \tau)\right)
$$

and

$$
\begin{aligned}
& v^{-\frac{1}{2}} E^{* \prime}\left(g_{\tau}, 0, \Phi^{-}\right) \\
& =h_{q}\left(\log q v+2 \frac{\Lambda^{\prime}(1, \epsilon)}{\Lambda(1, \epsilon)}+\sum_{j=1}^{k} \frac{1}{j}\right)-2 \sum_{n>0} a_{n} p_{k}(4 \pi n v) e(n \tau) \\
& \quad-2 \sum_{n<0} \rho(-n) q_{k}(-4 \pi n v) e(n \tau) .
\end{aligned}
$$

Proof. First we observe that

$$
\prod_{p \nmid q \infty} \rho_{p}(d)\left(1 \pm \epsilon_{q}(d)\right)=\rho(|d|)\left(1 \pm \epsilon_{q}(d)\right)=2 \rho(d)
$$

since

$$
1=\prod_{p \leq \infty} \epsilon_{p}(d)=\operatorname{sign}(d) \epsilon_{q}(d) \prod_{p \mid d}(-1)^{\operatorname{ord}_{p} d} .
$$

Formula (2.8) is a special case of the Siegel-Weil formula. We give a direct proof here using Lemmas 2.1-2.3. First, the lemmas imply $E_{d}^{*}\left(g_{\tau}, 0, \Phi^{+}\right)=0$ unless $d \geq 0$ is an integer. When $d>0$ is an integer, the lemmas and (2.10) imply

$$
\begin{aligned}
E_{d}^{*}\left(g_{\tau}, 0, \Phi^{+}\right) & =q^{\frac{1}{2}} \prod_{p \nmid q \infty} \rho_{p}(d) \frac{1+\epsilon_{q}(d)}{\sqrt{-q}} 2 i v^{\frac{1}{2}} p_{k}(4 \pi d v) e(d \tau) \\
& =4 v^{\frac{1}{2}} \rho(d) p_{k}(4 \pi d v) e(d \tau) .
\end{aligned}
$$

The same lemmas also imply

$$
\begin{aligned}
E_{0}^{*}\left(g_{\tau}, 0, \Phi^{+}\right) & =q^{\frac{1}{2}} \Lambda(1, \epsilon) \Phi^{+}\left(g_{\tau}, 0\right)+q^{\frac{1}{2}} M^{*}(0) \Phi^{+}\left(g_{\tau}, 0\right) \\
& =h v^{\frac{1}{2}}+\Lambda(0, \epsilon) v^{\frac{1}{2}} \\
& =2 h v^{\frac{1}{2}}
\end{aligned}
$$

This proves (2.8).

As for (2.9), we again check term by term, and it is clear from the lemmas that $E_{d}^{*^{\prime}}\left(g_{\tau}, 0, \Phi^{-}\right)=0$ unless $d$ is an integer, which we assume from now on.

When $d<0, W_{d, \infty}^{*}\left(g_{\tau}, 0, \Phi^{-}\right)=0$ by Lemma 2.3(3), and so (using Lemmas 2.1-2.3 and (2.10))

$$
\begin{aligned}
E_{d}^{* \prime}\left(g_{\tau}, 0, \Phi^{-}\right) & =q^{\frac{1}{2}} W_{d, \infty}^{* \prime}\left(g_{\tau}, 0, \Phi_{\infty}\right) W_{d, q}^{*}\left(1,0, \Phi_{q}^{-}\right) \prod_{p \nmid q \infty} W_{d, p}^{*}\left(1,0, \Phi_{p}\right) \\
& =-2 v^{\frac{1}{2}} q_{k}(-4 \pi d v) e(d \tau)\left(1-\epsilon_{q}(d)\right) \prod_{p \nmid q \infty} \rho_{p}(d) \\
& =-2 v^{\frac{1}{2}} \rho(-d) q_{k}(-4 \pi d v) e(d \tau),
\end{aligned}
$$

as desired.

When $d>0$ and $\epsilon_{q}(d)=1$, one has $W_{d, q}^{*}\left(1,0, \Phi^{-}\right)=0$ and

$$
W_{d, q}^{* \prime}\left(1,0, \Phi^{-}\right)=\frac{-1}{\sqrt{-q}}\left(\operatorname{ord}_{q} d+1\right) \log q .
$$


The same computation using Lemmas 2.1-2.3 and (2.10) yields

$$
\begin{aligned}
E_{d}^{* \prime}\left(g_{\tau}, 0, \Phi^{-}\right) & =-2 v^{\frac{1}{2}} p_{k}(4 \pi d v) e(d \tau)\left(\operatorname{ord}_{q} d+1\right) \rho(d) \log q \\
& =-2 v^{\frac{1}{2}} a_{n} p_{k}(4 \pi d v) e(d \tau),
\end{aligned}
$$

since $a_{n}=\left(\operatorname{ord}_{q} d+1\right) \rho(d) \log q$ in this case.

When $d>0$ and $\epsilon_{q}(d)=-1$, there is a prime $l \mid d$ such that $W_{d, l}^{*}\left(1,0, \Phi_{l}\right)=$ $\rho_{l}(d)=0$ by $(2.10)$. In this case,

$$
W_{d, l}^{* \prime}\left(1,0, \Phi_{l}\right)=\frac{1}{2}\left(\operatorname{ord}_{l} d+1\right) \log l .
$$

The same calulation yields

$$
E_{d}^{* \prime}\left(g_{\tau}, 0, \Phi^{-}\right)=-2 v^{\frac{1}{2}} a_{n} p_{k}(4 \pi d v) e(d \tau),
$$

as desired.

Finally, when $d=0$, one has by the same lemmas,

$$
E_{0}^{*}\left(g_{\tau}, s, \Phi^{ \pm}\right)=\frac{1}{\prod_{j=1}^{k}\left(j+\frac{s}{2}\right)}(G(s) \pm G(-s))
$$

with

$$
G(s)=(q v)^{\frac{1+s}{2}} \Lambda(1+s, \epsilon) \prod_{j=1}^{k}\left(j+\frac{s}{2}\right) .
$$

So

$$
E_{0}^{* \prime}\left(g_{\tau}, 0, \Phi^{-}\right)=\frac{2 G^{\prime}(0)}{k !}=h v^{\frac{1}{2}}\left(\log (q v)+2 \frac{\Lambda^{\prime}(1, \epsilon)}{\Lambda(1, \epsilon)}+\sum_{j=1}^{k} \frac{1}{j}\right) .
$$

This finishes the proof of (2.9).

Proof of Theorem 0.1. One has by Proposition 2.4,

$$
E^{*}(\tau, 0)=\frac{1}{2} v^{-k-\frac{1}{2}} E^{*}\left(g_{\tau}, 0, \Phi^{+}\right)
$$

and

$$
E^{* \prime}(\tau, 0)=\frac{1}{2} v^{-k-\frac{1}{2}}\left[E^{* \prime}\left(g_{\tau}, 0, \Phi^{-}\right)-\frac{1}{2} \sum_{j=1}^{k} \frac{1}{j} E^{*}\left(g_{\tau}, 0, \Phi^{+}\right)\right] .
$$

Now Theorem 0.1 easily follows from Propositions 1.1 and 2.4 and Theorem 2.5.

\section{Proof of Theorem 0.2}

By Proposition 1.1 and Formulas (2.16) and (2.17), Theorem 0.2 is equivalent to the identity

$$
\left(\frac{|\tau|}{\tau}\right)^{2 k+1}\left(\begin{array}{l}
E^{*}\left(g_{-\frac{1}{q \tau}}, 0, \Phi^{+}\right) \\
E^{* \prime}\left(g_{-\frac{1}{q \tau}}, 0, \Phi^{-}\right)
\end{array}\right)=i\left(\begin{array}{cc}
-1 & 0 \\
\frac{1}{2} \log q & 1
\end{array}\right)\left(\begin{array}{c}
E^{*}\left(g_{\tau}, 0, \Phi^{+}\right) \\
E^{* \prime}\left(g_{\tau}, 0, \Phi^{-}\right)
\end{array}\right) .
$$

To prove (3.1), one observes the following trivial but fundamental identity and computes both sides:

$$
E^{*}\left(w_{\infty}^{-1} g_{q \tau}, s, \Phi^{ \pm}\right)=E^{*}\left(w_{f} g_{q \tau}, s, \Phi^{ \pm}\right) .
$$


Here $w_{f}$ and $w_{\infty}$ are the images of $w=\left(\begin{array}{cc}0 & -1 \\ 1 & 0\end{array}\right)$ in $G\left(\mathbb{A}_{f}\right)$ and $G(\mathbb{R})$ respectively. The left-hand side of this identity is given by

\section{Lemma 3.1.}

$$
E^{*}\left(w_{\infty}^{-1} g_{\tau}, s, \Phi^{ \pm}\right)=\left(\frac{|\tau|}{\tau}\right)^{2 k+1} E^{*}\left(g_{-\frac{1}{\tau}}, s, \Phi^{ \pm}\right)
$$

Proof. Write $w_{\infty}^{-1} g_{\tau}=g_{-\frac{1}{\tau}} k_{\theta}$; then $e^{i \theta}=|\tau| / \tau$. So one has, for any $\gamma \in G(\mathbb{Q})$,

$$
\Phi_{\infty}\left(\gamma_{\infty} w_{\infty}^{-1} g_{\tau}, s\right)=\left(\frac{|\tau|}{\tau}\right)^{2 k+1} \Phi_{\infty}\left(\gamma_{\infty} g_{-\frac{1}{\tau}}, s\right)
$$

Plugging this into the definition of the Eisenstein series, one gets the lemma.

For the right-hand side of (3.2), one has

\section{Lemma 3.2.}

$$
\left(\begin{array}{c}
E^{*}\left(w_{f} g_{q \tau}, 0, \Phi^{+}\right) \\
E^{* \prime}\left(w_{f} g_{q \tau}, 0, \Phi^{-}\right)
\end{array}\right)=i\left(\begin{array}{cc}
-1 & 0 \\
\frac{1}{2} \log q & 1
\end{array}\right)\left(\begin{array}{c}
E^{*}\left(g_{\tau}, 0, \Phi^{+}\right) \\
E^{* \prime}\left(g_{\tau}, 0, \Phi^{-}\right)
\end{array}\right) .
$$

Proof. We verify these identities by comparing the Fourier coefficients $E_{\frac{d}{q}}^{*}\left(w_{f} g_{q \tau}\right.$, $\left.s, \Phi^{ \pm}\right)$with $E_{d}^{*}\left(g_{\tau}, s, \Phi^{ \pm}\right)$. We may assume that $d$ is an integer by Lemmas 2.1-2.3. Straightforward calculation using the same lemmas yields, for any integer $d$,

$$
W_{\frac{d}{q}, p}^{*}\left(w_{f} g_{q \tau}, s, \Phi^{ \pm}\right)=F_{p}^{ \pm}(d) W_{d, p}^{*}\left(g_{\tau}, s, \Phi^{ \pm}\right)
$$

with

$$
F_{p}^{ \pm}(d)= \begin{cases}1 & \text { if } p \nmid q \infty \\ q^{\frac{1-s}{2}} & \text { if } p=\infty \\ \pm \frac{1}{\sqrt{-q}} \frac{1 \pm \epsilon_{q}(d) q^{-s r}}{1 \pm \epsilon_{q}(d) q^{-s(r+1)}} & \text { if } p=q .\end{cases}
$$

Here $r=\operatorname{ord}_{q} d$. We will verify the derivative part and leave the value part to the reader. First assume $d \neq 0$. It follows from (3.3) that

$$
E_{\frac{d}{q}}^{* \prime}\left(w_{f} g_{q \tau}, 0, \Phi^{-}\right)=i E_{d}^{* \prime}\left(g_{\tau}, 0, \Phi^{-}\right) \begin{cases}1 & \text { if } \epsilon_{q}(d)=-1 \\ 1-\frac{1}{\operatorname{ord}_{q} d+1} & \text { if } \epsilon_{q}(d)=1\end{cases}
$$

When $\epsilon_{q}(d)=1$, one has by (2.11) and (2.12),

$$
E_{d}^{* \prime}\left(g_{\tau}, 0, \Phi^{-}\right)=-E_{d}^{*}\left(g_{\tau}, 0, \Phi^{+}\right) \frac{\operatorname{ord}_{q} d+1}{2} \log q
$$

So

$$
E_{\frac{d}{q}}^{* \prime}\left(w_{f} g_{q \tau}, 0, \Phi^{-}\right)=i E_{d}^{* \prime}\left(g_{\tau}, 0, \Phi^{-}\right)+\frac{i}{2} \log q E_{d}^{*}\left(g_{\tau}, 0, \Phi^{+}\right),
$$

as desired. When $\epsilon_{q}(d)=-1$ we have $E_{d}^{*}\left(g_{\tau}, \phi 0, \Phi^{+}\right)=0$, and (3.5) still holds.

It remains to check the constant term. Recall (2.13)-(2.15). Direct calculation using Lemmas 2.1-2.3 also gives

$$
E_{0}^{*}\left(w_{f} g_{q \tau}, s, \Phi^{ \pm}\right)=\mp \frac{i}{\prod_{j=1}^{k}\left(j+\frac{s}{2}\right)}\left(q^{\frac{s}{2}} G(s) \pm q^{\frac{-s}{2}} G(-s)\right) .
$$


Therefore,

$$
\begin{aligned}
E_{0}^{* \prime}\left(w_{f} g_{q \tau}, 0, \Phi^{-}\right) & =i \frac{2 G^{\prime}(0)}{k !}+i \frac{2 G(0)}{k !} \frac{1}{2} \log q \\
& =i E_{0}^{* \prime}\left(g_{\tau}, 0, \Phi^{-}\right)+\frac{i}{2} \log q E_{0}^{*}\left(g_{q \tau}, 0, \Phi^{+}\right),
\end{aligned}
$$

as expected, too.

\section{4. $L$-SERIES}

Recall that $q$ is a prime congruent to 3 modulo 4 and $\boldsymbol{k}=\mathbb{Q}(\sqrt{-q})$ is the associated imaginary quadratic field. Recall also ( $\underline{\mathrm{Roh}})$ that a canonical Hecke character of $\boldsymbol{k}$ of weight $2 k+1$ is a Hecke character $\mu$ satisfying

(1) The conductor of $\mu$ is $\sqrt{-q} \mathcal{O}_{\boldsymbol{k}}$.

(2) $\quad \mu(\overline{\mathfrak{A}})=\overline{\mu(\mathfrak{A})}$ for an ideal $\mathfrak{A}$ relatively prime to $\sqrt{-q} \mathcal{O}_{\boldsymbol{k}}$.

(3) $\mu\left(\alpha \mathcal{O}_{\boldsymbol{k}}\right)= \pm \alpha^{2 k+1}$.

In this section, we will give an explicit formula for the central derivative of its $L$-function, which has deep arithmetic implications as mentioned in the introduction. We refer to Gro for the arithmetics of elliptic curves associated to these Hecke characters (see also [MY] and $[\mathrm{Ya}$ and the reference there for more recent developments). For each ideal class $C$ of $\boldsymbol{k}$, we can define the partial $L$-series by

$$
L(s, \mu, C)=\sum_{\mathfrak{B} \in C, \text { integral }} \mu(\mathfrak{B})(N \mathfrak{B})^{-s} .
$$

Of course, $L(s, \mu)=\sum_{C \in \mathrm{CL}(\boldsymbol{k})} L(s, \mu, C)$. The following proposition is standard.

Proposition 4.1. Let $\mathfrak{A} \in C$ be a primitive ideal of $\boldsymbol{k}$ relatively prime to $2 q$, and write

$$
\mathfrak{A}=\left[a, \frac{b+\sqrt{-q}}{2}\right], \quad \text { with } a>0, b \equiv 0 \quad \bmod q .
$$

Let $\tau_{\mathfrak{A}}=\frac{b+\sqrt{-q}}{2 a q}$. Then

$$
L(s+k+1, \mu, C)=\frac{\mu(\mathfrak{A})}{(N \mathfrak{A})^{2 k+1}}(2 \sqrt{q})^{s-k} L(2 s+1, \epsilon) E\left(\tau_{\mathfrak{A}}, 2 s\right) .
$$

Set

$$
\theta_{k}(\tau)=h+2 \sum_{n>0} \rho(n) p_{k}(4 \pi n v) e(n \tau)
$$

and

$$
\phi_{k}(\tau)=a_{0}(v)-2 \sum_{n>0} a_{n} p_{k}(4 \pi n v) e(n \tau)-2 \sum_{n<0} \rho(-n) q_{k}(-4 \pi n v) e(n \tau) .
$$

Then Theorem 0.1 says that

$$
E^{*}(\tau, 0)=v^{-k} \theta_{k}(\tau)
$$

and

$$
E^{* \prime}(\tau, 0)=\frac{1}{2} v^{-k}\left(\phi_{k}(\tau)-\frac{1}{2} \sum_{j=1}^{k} \frac{1}{j} \theta_{k}(\tau)\right) .
$$


Corollary 4.2. Let the notation be as in Proposition 4.1.

(1) The central L-value is

$$
L(k+1, \mu, C)=\frac{\pi \mu(\mathfrak{A})}{\sqrt{q}(N \mathfrak{A})^{k+1}} \theta_{k}\left(\tau_{\mathfrak{A}}\right) .
$$

(2) When the root number of $\mu$ is -1 , i.e., $(-1)^{k}\left(\frac{2}{q}\right)=-1$, the central $L$ derivative

$$
L^{\prime}(k+1, \mu, C)=\frac{\pi \mu(\mathfrak{A})}{\sqrt{q}(N \mathfrak{A})^{k+1}} \phi_{k}\left(\tau_{\mathfrak{A}}\right) .
$$

In particular,

$$
\begin{aligned}
& L^{\prime}(k+1, \mu, \text { trivial }) \\
& =\frac{\pi}{\sqrt{q}} \phi_{k}\left(\frac{1}{2}+\frac{i}{2 \sqrt{q}}\right) \\
& =\frac{\pi}{\sqrt{q}}\left[h\left(\log \frac{\sqrt{q}}{2}+2 \frac{\Lambda^{\prime}(1, \epsilon)}{\Lambda(1, \epsilon)}+\sum_{j=1}^{k} \frac{1}{j}\right)\right. \\
& \left.\quad-2 \sum_{n>0}(-1)^{n} a_{n} p_{k}\left(\frac{2 \pi n}{\sqrt{q}}\right) e^{-\frac{\pi n}{\sqrt{q}}}-2 \sum_{n<0}(-1)^{n} \rho(-n) q_{k}\left(-\frac{2 \pi n}{\sqrt{q}}\right) e^{-\frac{\pi n}{\sqrt{q}}}\right] .
\end{aligned}
$$

Proof. Only the second one needs a little explanation. When $(-1)^{k}\left(\frac{2}{q}\right)=-1$ we have $L(k+1, \mu, C)=0$ automatically and thus $\theta_{k}\left(\tau_{\mathfrak{A}}\right)=0$. So Theorem 0.1 and Proposition 4.1 imply

$$
\begin{aligned}
L^{\prime}(k+1, \mu, C) & =\frac{\pi \mu(\mathfrak{A})}{\sqrt{q}(N \mathfrak{A})^{2 k+1}}(2 \sqrt{q})^{-k} 2 E^{* \prime}\left(\tau_{\mathfrak{A}}, 0\right) \\
& =\frac{\pi \mu(\mathfrak{A})}{\sqrt{q}(N \mathfrak{A})^{k+1}}\left(\phi_{k}\left(\tau_{\mathfrak{A}}\right)-\frac{1}{2} \sum_{j=1}^{k} \frac{1}{j} \theta_{k}\left(\tau_{\mathfrak{A}}\right)\right) \\
& =\frac{\pi \mu(\mathfrak{A})}{\sqrt{q}(N \mathfrak{A})^{k+1}} \phi_{k}\left(\tau_{\mathfrak{A}}\right) .
\end{aligned}
$$

When $C$ is trivial, one can take $\mathfrak{A}=\mathcal{O}_{\boldsymbol{k}}$. In this case, $a=1$ and $\frac{b}{2 q} \equiv \frac{1}{2} \bmod 1$, and thus

$$
\phi_{k}\left(\tau_{\mathfrak{A}}\right)=\phi_{k}\left(\frac{1}{2}+\frac{i}{2 \sqrt{q}}\right) .
$$

In recent joint work with S. Miller ([MY $]$ ), we proved that $L^{\prime}(1, \mu$, trivial $)>0$ when $q \equiv 3 \bmod 8$ and $k=0$. Combining that with Corollary 4.2 , one has the following curious inequality:

$$
\begin{aligned}
& h\left(\log \frac{\sqrt{q}}{2}+2 \frac{\Lambda^{\prime}(1, \epsilon)}{\Lambda(1, \epsilon)}+\sum_{j=1}^{k} \frac{1}{j}\right) \\
& >2 \sum_{n>0}(-1)^{n} a_{n} p_{k}\left(\frac{2 \pi n}{\sqrt{q}}\right) e^{-\frac{\pi n}{\sqrt{q}}}+2 \sum_{n<0}(-1)^{n} \rho(-n) q_{k}\left(-\frac{2 \pi n}{\sqrt{q}}\right) e^{-\frac{\pi n}{\sqrt{q}}} .
\end{aligned}
$$




\section{ACKNOWLEDGEMENT}

This work was inspired by joint work with Steve Kudla and Michael Rapoport. The author thanks them for the inspiration. He thanks Rene Schoof for numerically verifying the formulae in Corollary 4.2 , which corrects a mistake in an earlier version of this paper. Finally, he thanks Dick Gross, Steve Miller, and David Rohrlich for stimulating discussions.

\section{REFERENCES}

[Gro] B. Gross, Arithmetic on elliptic curves with complex multiplication, Lecture Notes in Math., no. 776, Springer-Verlag, Berlin, 1980. MR 81f:10041

[GZ] B. Gross and D. Zagier, Heegner points and derivatives of L-series, Invent. Math. 84 (1986), 225-320. MR 87j:11057

$[\mathrm{Ku}] \quad$ S. Kudla, Central derivatives of Eisenstein series and height pairings, Ann. Math. 146 (1997), 545-646. MR 99j:11047

[KRY] S. Kudla, M. Rapoport, and T.H. Yang, On the derivative of an Eisenstein series of weight one, Internat. Math. Res. Notices 7 (1999), 347-385. MR 2000b:11057

[MY] S. D. Miller and T. H. Yang, Non-vanishing of the central derivative of canonical Hecke L-functions, Math. Res. Letters 7 (2000), 263-277. MR 2001i:11058

[Roh] D. Rohrlich, Root numbers of Hecke L-functions of CM fields, Amer. J. Math. 104 (1982), 517-543. MR 83j:12011

[Sh] G. Shimura, Confluent hypergeometric functions on tube domains, Math. Ann. 260 (1982), 269-302. MR 84f:32040

[Ya] T.H. Yang, On CM abelian varieties over imaginary quadratic fields, preprint.

Department of Mathematics, University of Wisconsin, Madison, Wisconsin 53706

E-mail address: thyang@math.wisc.edu 